\title{
sciendo
}

Transport and Telecommunication, 2019, volume 20, no. 2, 133-141

Transport and Telecommunication Institute, Lomonosova 1, Riga, LV-1019, Latvia

DOI 10.2478/ttj-2019-0012

\section{SIMULATION MODEL OF TRANSPORT TRACEABILITY SYSTEM FOR TRANSIT CUSTOMS GOODS WITHIN THE EEU}

\author{
Daulet Akhmedov $^{1}$, Denis Yeryomin ${ }^{2}$, Dinara Zhaxygulova ${ }^{3}$, Sergey Trepashko ${ }^{4}$ \\ 1,2,3,4 AALR "Institute of Space Techniques and Technologies" \\ Almaty, Kazakhstan, Kislovodskiy str., 34 \\ ${ }^{1}$ lacp@mail.ru, ${ }^{2}$ denis.e@bk.ru, ${ }^{3}$ zhaxygulova@istt.kz, ${ }^{4}$ trepasko.sr@gmail.com
}

This article reviews the transport traceability system for customs goods within the Eurasian Economic Union, which is the automated data system used to control integrity and movement of cargo section of a carrier vehicle by means of satellite navigation system, mobile cellular communication system and satellite communication system. Article is dedicated to the development of a model that simulates functions of transport traceability system for customs goods, which are being transported using the international transit transport corridors, and this model would be also used for assessment of a demand on electronic seals by seal installation stations, and for determination of correspondence between simulated transport route and actual travel time. Simulation model was already used in a test project, where it was integrated into "EVAC Transit" transport traceability system, and it has proven it's operability and efficiency.

Keywords: simulation model, traffic stream, traceability system, electronic seal, international transit transport corridor, smart transport system

\section{Introduction}

By the decision of heads of the Commonwealth of Independent States (CIS countries), the united transport traceability system for customs goods that are transported via international transport corridors of the customs union of Eurasian Economic Union (EEU) is to be used for "green corridor" transit across the EEU territory. The United Transport Traceability System for Customs Goods (UTTSCG) across the EEU territory is the automated system used for control over integrity and movement of cargo section of a vehicle by means of satellite navigation system, mobile cellular communication system and satellite communication system. The UTTSCG across the EEU should be based on the integration of national systems of monitoring over customs goods of EEU customs union members. The purpose of implementation of the UTTSCG system is in the increase of efficiency of transhipment across the EEU territory due to the shortening of time required to cross the EEU route. The UTTSCG is created in order to solve the following tasks:

- Control of import, export and transit of customs goods (including products under export control and supervision: weapons and military vehicle, uranium products, explosive and chemical substances);

- Suppression of illegal traffic;

- Cancelation of inspection and screening of vehicle within the EEU;

- Exemption from customs convoy service;

- Exemption from collateral security (deposit) for transported goods;

- Online transmission of data to goods owner about the location of transport;

- Provision of unrestricted passage of customs goods through system of risks management (auto validation) in case there are no violations in terms of the mode of transportation of customs goods (deviations from planned route and travel schedule). Risks management system provides efficient control due to data obtained from UTTSCG system.

National UTTSCG system "EVAC Transit" that is used within the Kazakhstan territory includes the following components:

- Software for control centre;

- Software for automated workplace for system operator;

- Main data processing Center (DPC);

- Secondary (reserve) DPC;

- Integrative portal for EEU member countries.

The role of a sensor for united transport traceability system within EEU and customs goods traceability system within EEU, and the source of reference data is played by the electronic lock-and-seal device. 
According to the order No. 374 issued on March 15, 2018 by the Minister of Finance of the Republic of Kazakhstan, electronic lock-and-seal device is acknowledged to be one of the identification means of state revenue bodies, which are installed by authorized representatives of state revenue bodies onto the locking mechanisms of cargo sections of the transport vehicle when transporting goods that are under the customs supervision. Electronic lock-and-seal device (electronic seal) is intended to perform the following functions:

- Online satellite monitoring of goods location (cargo section);

- Transmit alarm signal in case of loss of integrity (damage or distraction) of electronic seal;

- Transmit alarm signal in case of separation of cargo section from the actual transport;

- Transmit alarm signal in case of going off the coordinated and accepted route and in case of not following the travel time schedule.

UTTSCG "EVAC Transit" provides monitoring of movement of transport and determination of goods location, as well as assessment of correspondence of the actual travel route with the planned route, control of goods integrity and safety, and control of customs goods transportation mode. The role of a sensor that determines the location of goods is played by electronic seals - on-board terminals used for collection and transmission of data (to Data Processing Center) and connected to the door-locking device. Electronic seals are installed at terminal installation stations (initial point of the route) onto doors of the cargo section of a transport, and then installed seals are being registered in the system, linking them to a license plate of a vehicle. Electronic seal reads location coordinates of goods during the whole time of travel, from the starting point of a route to it's end, and transmits data to system online, which allows to draw the trajectory of movement and time schedule, and to control the correspondence of travel to the planned route. This seal can also transmit alarm signals to system in case of unauthorized removal of a seal (destruction of locking mechanism), which means the violation of customs goods transportation mode. Authorized (non-destructive) removal of seal is only possible at terminals installation and removal stations along with registration of a corresponding procedure in system.

System of satellite monitoring over the goods use the following on-board navigation-connected terminals for determination of location: on-board terminal, on-board tracker with satellite navigation, electronic seals (locking device) with satellite navigation. No matter what type of the device is used, all of them provide measurements of coordinates, defining the location of transport with goods using signals from satellite navigation systems and online transmission of data to the DPC using GSM mobile communication channels or IRIDIUM satellite communication channels.

\section{Review of Literature}

On stage of realization of transport traceability for transit goods, it is necessary to pre-define the demand on electronic seals at installation stations and practicability of modes of customs goods transportation by means of a vehicle. In order to provide efficient method of research of transporting system processes, the simulation modelling is used. It gives opportunity to perform dedicated research of a structure and functions of the actual complex process and to perform optimization of some of it's parameters.

Simulation model of UTTSCG for EEU is intended for calculation of the necessary amount of electronic seals for satisfaction of demand at seals installation station and conformation of correspondence with planned route across national and international transit corridors, as well as correspondence of planned travel schedule with the actual time of travel.

Actual processes within transport systems are of the "analogue" character, and in order to simulate them in digital calculations, the sampling of process parameters is required in terms of the characteristic with the set time intervals, which means "digitization" of analogue parameters and their transformation into discrete readings. Nowadays, there are several models being used with similar algorithms, yet they differ in terms of a discretion mode, due to which they have different operational qualities.

Transporting task is the serious problem from perspective of practical use of simulation tools. It requires involvement of formal approach to analysis and forecast of characteristics of dynamic systems (Nechayev et al., 2011) and traffic in particular, technologies of calculation experiment performance (Nechayev, 2012; Vasilyev and Osipov, 2011). Researches on simulation of traffic streams (Cremer and May, 1986; Nagel and Schreckenberg, 1992; Kesting et al., 2007, 2010; Trapeznikova et al., 2011) reflects the necessity in development of information technologies that correspond to the level of presents tasks.

The goal of simulation modelling is connected with simulation of traffic streams and consideration of interaction among members of traffic and systems of traffic control. Research of traffic stream parameters allows to develop practical ways of control without performing long-term and expensive real-life experiments.

Cellular automation model by Nagel-Schreckenberg - microscopic model of transport streams based on discretion of time and space - is reviewed as one of the simulation methods. These models are 
interesting, first of all, due to their fast operation and complex behaviour in dynamics, including such phenomena as self-organizing criticality, formation of spiral samples, fluctuating or chaotic order of conditions. High speed and efficiency of calculations in combination with the following properties make cellular automation models ideal for parallel calculations:

- Discretion of space into identical cells (grid points $j$ ) that have size of $\Delta x$;

- Final amount of possible conditions $g(x)$;

- Parallel update of cells in time $t=i \cdot \Delta t$ with common step of $t$;

- Globally used rules of cells update;

- Interaction of close order with final amount of neighbouring points.

Models of cellular automation are used for modelling of moving loose granular medium and liquids when calculating the process of a chemical reaction, or when simulating an avalanche. Cellular automation has found it's use in simulation of traffic dynamics due to tasks of mathematical analysis of traffic instability that leads to "break-up" rhythm of movement (start-stop) during traffic jams. Cellular automation describes dynamic processes in less detail than a leader-following model, however, their more simple structure allows to rapidly calculate the movement of large amount of vehicles. In Nagel and Schreckenberg (1992) a cellular automation model of Nagel-Schreckenberg, the highway is divided into cells $j$ that have a length of $\Delta x$, and the time $t$ per $i$ intervals that equal $\Delta t=1$ second (discretion interval). Each cell is either empty or contains exactly one single vehicle that moves with a speed of $v_{i}$ in accordance to the formula below (1):

$v_{i}=\hat{v}_{i} \frac{\Delta x}{\Delta t}$

where, $v_{i}$-speed of a vehicle at the given moment of time $i$;

$\hat{v}_{i} \in\left\{0,1,2, \ldots, \hat{v}_{\max }\right\}$ - dimensionless scalable (discrete) speed of a vehicle in a given moment of time $i, \hat{v}_{i}$ may be equal to one of the values from 0 to $\hat{v}_{\max }$;

$\frac{\Delta x}{\Delta t}$ - discretion interval of vehicle speed, which physically means the speed of passing one cell of a route with a length of $\Delta x$ per one discretion interval $\Delta t=1$ second.

In case of a highways it is the practice to choose the size of elementary cell being equal to $\Delta x=7,5$ meters, and the speed of free movement $v_{0}=120-140 \mathrm{~km} /$ hour, then the discretion interval of speed is equal to $\frac{\Delta x}{\Delta t}=7.5 \mathrm{~m} / \mathrm{sec}=27 \mathrm{~km} / \mathrm{h}$, and the maximum discrete speed is equal to $\hat{v}_{\max }=v_{0} \frac{\Delta t}{\Delta x}=5$ (which corresponds to maximum speed of free movement of $135 \mathrm{~km} / \mathrm{h}$ ).

In cellular automation model, positions of vehicles are updated simultaneously with discretion interval $\Delta t$, following the given rules:

- Rule 1 "Movement": Vehicle moves in each following moment of time $i$ (with discretion interval of $\Delta t$ ) per $\hat{v}_{i}$ cells.

- Rule 2 “Acceleration": if the vehicle has not reached it's maximum speed $\hat{v}_{\max }$, then it should increase it's speed up to $\hat{v}_{i+1}=\left(\hat{v}_{i}+2\right)$ with constant acceleration of $\Delta x /(\Delta t)^{2}$.

- Rule 3 "Deceleration": if the distance (amount of empty cells $\hat{d}_{i}$ ) to the vehicle in front is not greater than $d_{i} \leq \hat{v}_{i}$, the speed of a vehicle should be decrease by one unit to $\hat{v}_{i+1}=\left(\hat{d}_{i}-1\right)$, otherwise the speed should not be changed $\hat{v}_{i+1}=\hat{v}_{i}$.

- Rule 4 "Random perturbations": of the speed of a vehicle is not equal to zero $\hat{v}_{i}>0$, then it might be decreased by one unit with deceleration probability of $p$ to $\hat{v}_{i+1}=\left(\hat{v}_{i}-1\right)$. Zero speed is not changing $\hat{v}_{i+1}=\hat{v}_{i}=0$. The deceleration probability $p$ in case of Nagel-Schreckenberg model describes fluctuations of vehicle speed due to delay of acceleration by driver (delayed movement, increase of distance to the vehicle in front). 
According to Rule 3, vehicle always moves with such a speed that does not exceed the opportunity provided by one free cells (at least one) in front, with a time difference of $\Delta t$. Thus, discretion interval $\Delta t$ simultaneously plays several roles: cells update interval, adaptation time $\tau$ and safety time $T$ (desired time gap). Discretion interval $\Delta t$ is coordinated with minimum required free space, which is the inversion of traffic jam density. Formula of speed movement and rules in above-mentioned model are exceptionally compact. In Nagel-Schreckenberg model the following values $p=0.5$ and $\hat{v}_{\max }=5$ (that equals to $135 \mathrm{~km} / \mathrm{h}$ ) are suggested for model of movement across highways, which means the dynamic movement with speed fluctuations. For the model of movement within a city, recommended parameters are $p=0.2$ and $\hat{v}_{\max }=\{2,3\}$ (that equals to 54 and $81 \mathrm{~km} / \mathrm{h}$ accordingly).

Intelligent Driver Model (IDM) by Treiber (Kesting et al., 2007, 2010) shows the most correct results of traffic streams modelling and the closest parameters to the actual driving on highways. Treiber's IDM model can be easily adjusted, does not cause emergency situations, model is numerically effective, shows realistic behaviour during acceleration (deceleration), leads to empirically observable data close to real-life tests. Control diagram of IDM model corresponds to obtained kinetic theory of traffic management model. It allows to combine micro and macro simulation of traffic streams. In other aspects, IDM shares the peculiarities of Nagel-Schreckenberg's cellular model.

In IDM the acceleration of a vehicle $\alpha$ is a constant function of speed $v_{0}$, and gap $s_{\alpha}=\left(d_{\alpha}-l_{\alpha-1}\right)$ between vehicle $\alpha$ and $\alpha-1$, the differences in speed $\Delta v_{\alpha}=\left(v_{\alpha}-v_{\alpha-1}\right)$ of the observed vehicle $\alpha$ and the other vehicle in front $\alpha-1$ :

$$
\frac{d v_{\alpha}}{d t}=a_{\alpha}\left[1-\left(\frac{v_{\alpha}}{v_{\alpha}^{0}}\right)^{\delta}-\left(\frac{s_{\alpha}\left(v_{\alpha}, \Delta v_{\alpha}\right)}{s_{0}}\right)^{2}\right],
$$

where parameters of IDM are identical to parameters of the "following a leader" model:

$v_{\alpha}$-speed of a vehicle $\alpha$;

$a_{\alpha}$ - maximum acceleration of a vehicle $\alpha$;

$v_{\alpha}^{0}$-speed of free movement of a vehicle $\alpha$;

$\delta$ - index of vehicle's acceleration rate $\alpha$;

$s_{0}-$ minimum distance of approach (gap) between vehicles.

In formula (2), the acceleration strategy on a traffic-free road $v_{\text {free }}(v)=a\left\lfloor 1-\left(v_{\alpha} / v_{0}\right)^{\delta}\right\rfloor$ combines with deceleration and braking strategy $v_{\text {brake }}(s, v, \Delta v)=-a\left(s_{\alpha} / s_{0}\right)^{2}$, which becomes predominate, if the gap $s_{\alpha}$ to the leading vehicle becomes less than the minimum safe gap $s_{0}$. Free movement is characterized by desired speed $v_{0}$, maximum acceleration $a$ and index of acceleration rate $\delta$. If $\delta=1$ then the exponential, in terms of time, acceleration takes place, if $\delta \rightarrow \infty$ the acceleration is performed with the maximum acceleration $a$. Parameters of IDM model $v_{0}, T, s_{0}, a, b$ have acceptable interpretation, they are justified, empirically measurable and have realistic values. Rated parameters of movement in simulation intelligent driver model by Triber are given in the Table 1 below (Kesting and Treiber, 2008; Babicheva et al., 2015).

Nagel-Schreckenberg's cellular automation model and intelligent driver model by Triber consider a vehicle as the minimal object of traffic, which reacts on environmental conditions such as speed of the vehicle itself, speed of neighbouring vehicles (in front, behind, and on lanes next to it).

Table 1. Parameters of movement for IDM by Triber

\begin{tabular}{|l|c|c|}
\hline Parameter & Passenger car & Goods-carrying vehicle \\
\hline Speed of free movement $v_{0}, \mathrm{~km} / \mathrm{h}$ & 120 & 85 \\
\hline Index of acceleration rate $\delta$ & 4 & 4 \\
\hline Safe (desired) time gap $T, \mathrm{sec}$ & 1.5 & 2.0 \\
\hline Minimal distance (minimal gap) $s_{0}, \mathrm{~m}$ & 2.0 & 4.0 \\
\hline Maximum acceleration $a, \mathrm{~m} / \mathrm{sec}^{2}$ & 1.4 & 0.7 \\
\hline Braking deceleration $b, \mathrm{~m} / \mathrm{sec}^{2}$ & 2.0 & 2.0 \\
\hline
\end{tabular}


Nagel-Schreckenberg's cellular automation model and intelligent driver model by Triber consider a vehicle as the minimal object of traffic, which reacts on environmental conditions such as speed of the vehicle itself, speed of neighbouring vehicles (in front, behind, and on lanes next to it).

In case of Nagel-Schreckenberg's cellular automation model, discretion by cells allows to speed up (hundreds of times faster) the process of simulation, because such discretion gives opportunity to use inner and outer parallelism of calculation systems efficiently. Inner parallelism lies in the fact that it is possible to process a group of cells within one operation. Outer parallelism lies in the fact that it is now becomes possible to use CUDA and OpenCL means for simulation of behaviour of separate cells, at the same time allowing to combine simulation of group of cells with MPI (Message Passing Interface) tool. All this gives opportunity to use calculation clusters efficiently and to achieve speed of online simulation even with large amount of tasks.

In Treiber's IDM the discretion by time does not have such properties. Using the time discretion for all vehicle, coordinates of each vehicle receive constant values, which forces operators to use more sophisticated methods (for example, connected lists) and more demanding resources of calculation system. In Nagel-Schreckenberg's model, in order to define vehicle's coordinates it is enough to have a block with known length for each driving lane. Unlike Nagel-Schreckenberg's model, parallel update of all positions of a vehicle in case of Treiber's method is possible only for non-connected roads. Complexity of algorithms of free space search in order to change lanes depends on the amount of vehicles present on the lane and increases along with increase of number of lanes.

Nagel-Schreckenberg's model is not possible to properly distinguish such different vehicles as 23 feet-long cargo truck and 6.5 feet-long motorcycle, though their behaviour on the road would, obviously, be completely different. Parameters of maximum acceleration and desired deceleration might be taken into consideration, but parameters of safe distance to the vehicle in front during lane change would be inevitably scaled to fit the size of a cell (typical 24.6 feet), and speed of different vehicle get scaled as well.

It is possible to avoid such issues if using Treiber's model. The safe distance to cargo truck is 13.1 feet, whereas for the passenger vehicle, such as car, safe distance would be 6.5 feet, yet the speed would be the same for both. Due to the fact that cargo transport prevails in traffic, it's behaviour should, and actually would differ from traffic with prevalence of cars. Traffic jams start to appear when traffic density is connected to the amount of cargo vehicles in completely non-linear pattern. It should be noted that moth methods, in their original state, are not applicable to highways with several lanes, complex crossroads and traffic circles.

\section{Traffic Simulation Methods}

Based on the given literature review and analysis of simulation models for transport systems, it is possible to make the following conclusions:

- for highways with intermediate density of traffic streams, with estimated rate of not more than $50-70 \%$ of traffic's maximum capacity, it would be enough to implement simulation cellular model created by Nagel-Schreckenberg, which has discretion by cells and would give an opportunity to realize speed simulation of traffics in online mode and with minimal expenses for calculation cluster;

- with increased requirements for accuracy of traffic streams simulation in cities and on highways, Treiber's IDM simulation model should be used, at the same time it is necessary to provide sufficient capacities and high operational speed of calculation cluster;

- for simulation of traffic streams on international transit transport corridors (ITTC) within the Republic of Kazakhstan, both Nagel-Schreckenberg's model and Treiber's IDM are applicable. Criteria for choice of method for simulation should be an assessment of maximum density of traffic streams. According to data of Statistics Committee of Ministry of national economy of the Republic of Kazakhstan, transportation of goods by means of automotive vehicles across the whole territory of Kazakhstan is equal to3 322.3 million tons in 2017, and it had various traffic density depending on the time of the year. If traffic density is under $70 \%$ of it's maximum traffic capacity, then it is enough to implement Nagel-Schreckenberg's cellular simulation model, if traffic density is exceeding $70 \%$, then Treiber's IDM should be used.

In order to research efficient management of traffic streams, it is necessary to create simulation model, which would solve several typical tasks. And due to the fact that there are no ideal model existing, which could have described all transport processes, there is a task on development and creation of simulation model that would be able to describe transport processes with sufficient level of adequacy for it's further practical use. It is impossible to define beforehand which algorithm from all the different 
algorithms used for simulation of traffic processes would provide results with set accuracy level, that's why future system should have the ability to solve one and the same tasks but using different methods. Comparison of results of mathematical calculations for various simulation models would give an opportunity for improvement of each model.

Transport processes simulation model consists of the following components:

- Informational component describes properties of simulation objects, such as characteristics of transport means and highway parameters, traffic streams intensity and much more;

- Calculation component defines the behaviour of objects within the given transport situation according to parameters of simulation objects. Calculation component allows the use of various mathematical models while having the same input data (traffic stream parameters);

- Visualization component reflects the process of transport streams simulation and it's results in a convenient and user-friendly way;

- Parallels creation component along with task module allows effective distribution of computational resources between separate sub-tasks that are solved in a process of execution (calculation) of mathematical model.

\section{Simulation Model of a Traffic Steam Traceability System}

Traffic streams simulation model applied for "EVAC Transit" goods traceability system was operating on $\mathrm{C} \#$ programming language (object-oriented language for development of applications based on Microsoft.NET Framework). Simulation gives opportunity to assess and optimize (distribute time and routes) cargo transport traffic within international transport corridors. Block-scheme for the algorithm of simulation model for traceability of transport traffic is shown on the Figure 1.

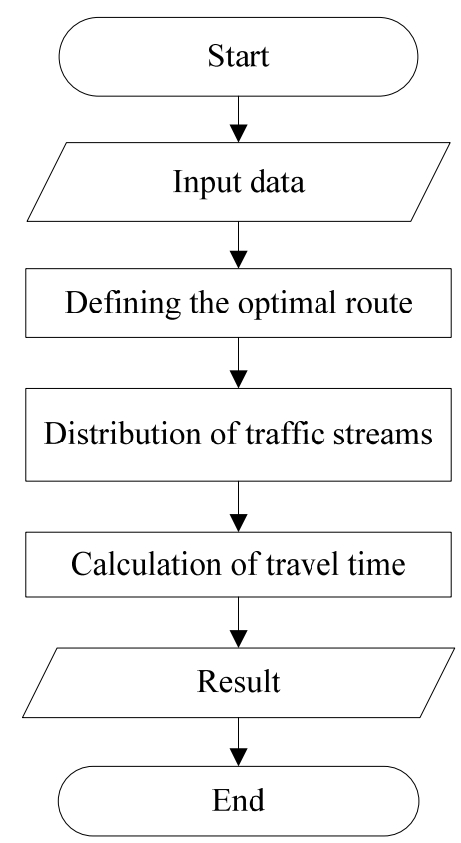

Figure 1. Block-scheme of algorithm for simulation model of traceability system of transport traffic

Block of input dynamic parameters consists of data about actual coordinates of goods (seals), obtained from all registered electronic seals online and updated with set frequency. Data on actual coordinates of goods are archived in a system and linked to numbers of electronic seals, data and time of fixation for the later comparison with results of traffic streams simulation model calculation and discover of mismatches.

There were initial setup parameters defined for simulation model (Table 2), used for mathematical models of transport streams of Nagel-Schreckenberg's cellular automation and Treiber's Intelligent Driver Model. Operator of simulation modelling system is able to change initial setup parameters with consideration of accumulated statistical data about parameters of traffic streams. 
Table 2. Initial setup parameters of simulation model

\begin{tabular}{|l|l|}
\hline Parameter of simulation model & Parameter's value \\
\hline Area of action of simulation model & The Republic of Kazakhstan \\
\hline Geometrical basis of simulation model & $\begin{array}{l}\text { Electronic digital map of the Republic of Kazakhstan with indication of } \\
\text { automotive roads and road infrastructure stations. }\end{array}$ \\
\hline Length of elementary road cell, $\Delta x, \mathrm{~m}$ & 20 \\
\hline Nominal length TC $l_{0}, \mathrm{~m}$ & 10 \\
\hline Discretion interval $\Delta t, \mathrm{sec}$ & 1 \\
\hline Nominal speed of movement $v_{0}, \mathrm{~km} / \mathrm{h}$ & 120 \\
\hline Index of acceleration degree $\delta$ & 4 \\
\hline Deceleration probability $p$ & 0,5 \\
\hline Nominal time gap $T, \sec$ & 2,0 \\
\hline Minimal distance to vehicle in front $s_{0}, \mathrm{~m}$ & 10,0 \\
\hline Maximum acceleration $a, \mathrm{~m} / \mathrm{sec}^{2}$ & 0,7 \\
\hline Maximum deceleration $b, \mathrm{~m} / \mathrm{sec}^{2}$ & 2,0 \\
\hline
\end{tabular}

There were statistical parameters defined for calculation of traffic streams simulation model, which may be updated by operator:

- Starting and ending points of route (electronic seal installation and removal stations - further referred as station);

- License plates of vehicles at electronic seals installation stations (amount of vehicles is captured automatically);

- Numbers of electronic seals installed and registered at installation stations (amount of seals, date and time of seals registration is captured automatically).

During the process of simulation modelling, users are able to define optimal route, distribute cargo transport traffics and calculate travel time. Optimal route is built based on highways of international (Fig. 2) and national (Fig. 3) importance, and based on minimal route length with indication of route length.

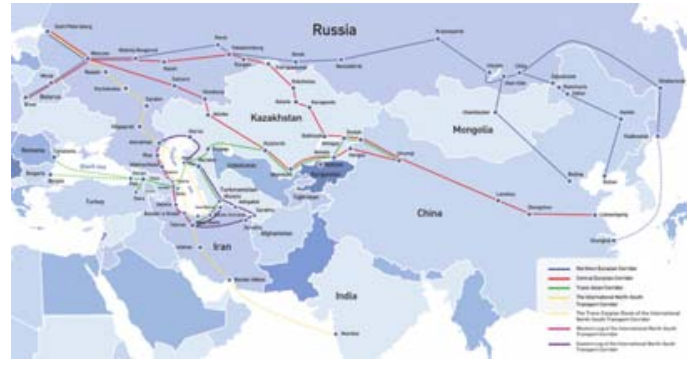

Figure 2. Map of main trans Eurasian transport corridors

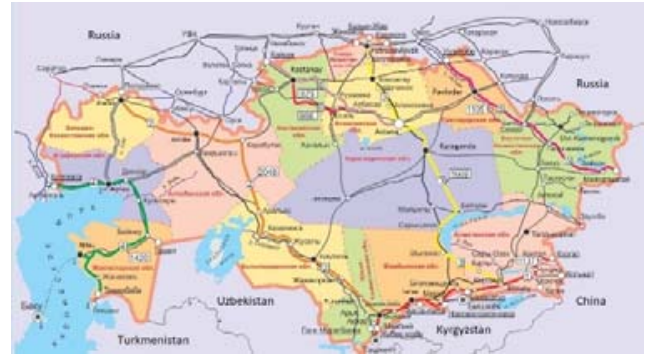

Figure 3. Map of ITTC and automotive roads in the Republic of Kazakhstan

During the process of calculation of simulation model, the route may be optimized with consideration of transport traffic density and travel schedule, and distribution of cargo transport streams is performed depending on volume and category of transported goods such as industrial and agricultural products, construction goods, trade network goods and so on. Calculation of travel time is performed in accordance with requirements of European agreement on operation of transport means crew that performs international transportation by road (ECTP) depending on travel distance, number of drivers, duration of driving and drivers' rest.

Results of simulation model for traffic streams are optimized distribution of routes and schedules, as well as amount of electronic seals (vehicles and goods) used per the designated period of time, diagram with the amount of vehicles passed through each electronic seals installation station.

Figure 4 shows the diagram for calculation of demand on electronic seals, results of which would be used later when providing installation stations with necessary amount for electronic seals for installation in cargo sections of transport at the starting point of the chosen route and for the given period of time. Figure 5 shows the diagram for the calculation of the amount of electronic seals that have arrived 
at the certain station at a given period of time. Data about the amount of electronic seals arrived at stations is necessary for distribution of load at stations, in case there are not so many vehicles coming out from the given seals installation/removal station.

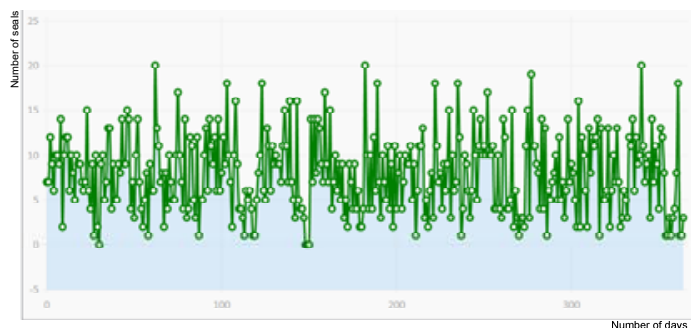

Figure 4. Diagram of demand on electronic seals at a given seals installation/removal station per the given period of time

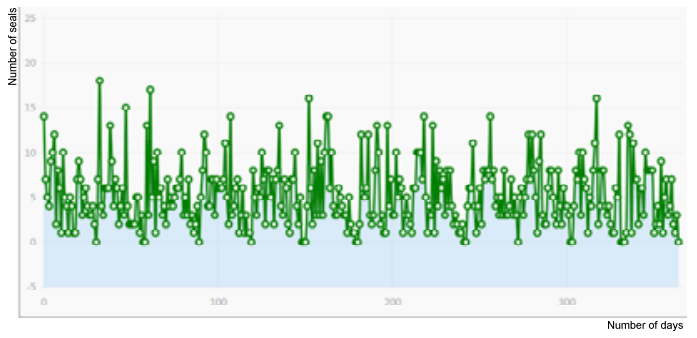

Figure 5. Diagram used for calculation of the amount of electronic seals that have arrived at installation/removal stations per given period of time

\section{Conclusion}

The designed simulation model was used as part of test projects of national electronic tracking system for customs goods "EVAC Transit", and played a role of assessing system for provision of necessary amount of electronic seals at installation stations and correspondence of simulated vehicle route and travel time with the actual movement mode. Within the period from April 25 till May 14 of the year 2018 , there was a test project of "EVAC Transit" national electronic tracking system of customs goods across the Kazakhstan, performed by employees of SLLP "Institute of Space technique and technology". During the period from September 19 till November 9 of the year 2018, there was a test project on monitoring of cargo transportation with the use of navigation seals in association with national operator of Russian Federation. Members of the test project the side of the Republic of Kazakhstan are SLLP "Institute of Space Technique and Technology" (national operator of "EVAC Transit" test project) and a Committee of State Revenues of the Republic of Kazakhstan, and from the side of Russian Federation OOO "RT-Invest Transport Systems" (national operator) and Federal customs service. Within this project there was performed a test of interaction mechanisms and information exchange between customs authorities that participate in a test project, and national operators of international transit cargo transportation tracking system for monitoring of goods that are transported in accordance with customs procedure of transit within the EEU territory.

During the performance of test project of customs goods electronic tracking system, "EVAC Transit" simulation model for transport traffic tracking has conformed it's operability and efficiency, and allowed to provide installation/removal stations with the necessary amount of electronic seals, which does not slow down the traffic, and allowed to discover violations of customs goods transportation mode (deviations from planned route and travel schedule), and timely react on destruction of locking devices of electronic seals.

\section{Acknowledgments}

This paper was performed as part of the BR05236762 project "Establishment of the hardware and software complex for controlling international transport transit corridors passing through the territories of the CIS member states on the basis of modern information and communication technologies" within financial management of projects program by Committee of Science and Education of the Ministry of Education and Science of the Republic of Kazakhstan.

\section{References}

1. Babicheva, T.S., Babichev, S.L. and Osipov, V.P. (2015) Architecture and methodological support of the module for simulation of transport processes within networking computer laboratory. Moscow: Preprints of IAM named after Keldish M.V.

2. Cremer, M. and May, A.D. (1987) An extended traffic flow model for inner urban freeways. In: Preprints of 5th IFAC/IFIP/IFORS International Conference on Control in Transportation Systems, July 1986, Vienna: IFAC Symposia Series, pp. 239-244. 
3. Kesting, A., Treiber, M. and Helbing, D. (2007) General lane-changing model mobil for carfollowing models. Transportation Research Record. №1999. pp. 86-94. DOI: 10.3141/1999-10.

4. Kesting, A., Treiber, M. and Helbing, D. (2010) Enhanced intelligent driver model to access the impact of driving strategies on traffic capacity. Philosophical Transactions of the Royal Society of London. №368. pp. 4585-4605. DOI: 10.1098/rsta.2010.0084.

5. Kesting, A. and Treiber, M. (2008) Calibrating car-following models by using trajectory data. Transportation Research Record. № 2088. pp. 148-156. DOI: 10.3141/2088-16.

6. Nagel, K., Schreckenberg, M. (1992) A cellular automaton model for freeway traffic. Journal de physique I. № 2 (12). pp. 2221-2229. DOI: 10.1051/jp1:1992277.

7. Nechayev, Y.I. (2012) Neurocomputers in intellectual technologies of XXI century. Moscow. Radio engineering.

8. Nechayev, Y.I., Lutin, A.V. (2011) Multi-agent technologies within complex adaptive systems of artificial intelligence. In: XIV International conference on soft calculations and measurements (SCM2011). June 2011, St. Petersburg ETU “LETI”, pp. 64-68.

9. Nechayev, Y.I., Osipov, V.P. (2011) Conceptual basis for creation of integrated calculated complex for support of decision making process. Neurocomputers: design, development, implementation. No.2, pp. 4-18.

10. Trapeznikova, M.A., Furmanov, I.R., Churbanova, N.G., Lipp, R. (2011) Simulation of multi-lane automotive traffic based on the theory of cellular automation. Mathematical modelling. No.6 (23), pp. 133-146.

11. Vasilyev, A.N., Osipov, V.P. (2011) Concept and principles of rational system of applied modelling and simulation. Neurocomputers. No.2, pp. 29-37. 